\title{
The Vibration of a Layered Rotating Planet and Bryan's Effect
}

\author{
Michael Y. Shatalov ${ }^{1}$, Stephan V. Joubert ${ }^{2}$ and Charlotta E. Coetzee ${ }^{2}$ \\ ${ }^{1}$ CSIR (Pretoria) and Tshwane University of Technology \\ ${ }^{2}$ Tshwane University of Technology \\ South Africa
}

\section{Introduction}

Because, among other seismological observations, it is important to be able to predict the location of the vibrating pattern of an earthquake, in this chapter we take the first tentative steps towards including "Bryan's effect" in a mathematical model for the seismic vibration pattern of a layered slowly rotating vibrating planet or moon.

Historically, G.H. Bryan observed (in his article (Bryan, 1890)) that when a vibrating structure is subjected to an inertial rotation, the vibrating pattern rotates within the structure at a rate proportional to (but in general not equal to) the inertial angular rate. This effect has come to be known as " Bryan's effect". It is interesting to note that (Rayleigh, 1894) mentions Bryan's effect in $\S 233$, but thereafter investigations of Bryan's effect appear to have lain dormant for about 75 years, reappearing in connection with a resonator gyroscope in 1965 in the small Delco Wakefield, MA, USA R\&D facility, according to (Rozelle, 2009). Rozelle states that the resonator gyroscope "has been utilized in many applications over its developmental lifetime: aircraft navigation, strategic missile navigation, underground borehole navigation, communication satellite stabilization, precision pointing, and in deep space missions". The effect may be useful in understanding the dynamics of pulsating stars in astrophysics and this was mentioned in the paper (Shatalov et al., 2009) in which the theoretical background on this chapter was discussed in general terms. For the constant of proportionality, Bryan made the following calculation for a body consisting of a ring or cylinder:

$$
B F=\frac{\text { Angular rate of the vibration pattern }}{\text { Inertial angular rate of the vibrating body }} .
$$

This constant of proportionality BF has come to be known as "Bryan's factor". The authors ( Zhuravlev \& Klimov, 1988) investigated Bryan's effect for an elastic, isotropic, spherically symmetric body, rotating in three-dimensional space. Among other results, they demonstrated that Bryan's factor depends on the vibration mode. Their concise formulation is given in general terms without computational detail or assumptions on the magnitude of rotation or illustrative examples. In (Shatalov et al., 2009) "slow rotation" (explained below) was assumed for spherical bodies consisting of concentric layers of elastic and/or acoustic media. In that paper, some detail was supplied for computations and an illustrative example was presented. They did not assume a "thin shell theory", as in (Loveday \& Rogers, 1998), 
where Bryan's effect is considered in a thin cylindrical shell for both high and low rotational rates. Consequently the model described by (Shatalov et al., 2009) is readily adaptable to the structure of an ideal planet or moon that is rotating slowly.

In this chapter we study Bryan's effect applied to a rotating planet or moon that may have several solid and liquid layers that are assumed to be rotating at the same rate. The rotation rate is assumed to be small when compared to the lowest eigenvalue of vibration $\omega=\frac{2 \pi}{T}$ where $T$ is the period of oscillation of the vibration pattern for a natural mode of vibration. As a first tentative approach, we discuss isotropic solid spherical layers that may be isotropically damped as well as isotropic inviscid spherical liquid layers. If damping is present in the media we expect to encounter, as a first approach, we assume that it will be isotropic and be light in the sense that the "damping factor" will be substantially smaller than the lowest eigenvalue of the system. Using Rayleigh's dissipation function (Goldstein, et al.), we demonstrate that light, isotropic, viscous damping does not influence Bryan's effect or Bryan's factor (Equation (1)). Hence, in the sequel, we assume that the body is subjected to nondecaying vibrations in one of its natural modes. The introduction of "impurities" such as prestress, mass-stiffness imperfections and anisotropic damping effects into the calculations is important for real-life situations (as opposed to ideal situations with at most isotropic, viscous damping) and has been earmarked by us for further study. Indeed, we have recently published a spherical model with varying mass density (Shatalov et al., 2011) that indicates that the rotation rate of the vibrating pattern is nonlinear (as opposed to the linear rate for the ideal state discussed below) and that this nonlinearity manifests itself as a "capture effect" whereby the rotation angle of the vibration pattern varies periodically.

\section{Preliminaries}

Consider a coordinate system $O x y z$ and a composite spherical body, with its centre at the origin $O$, consisting of concentric solid and or acoustic layers. Let $N$ be the number of concentric spherical media in the system and $a_{i}$ and $a_{i-1}$ the inner and outer radii of the $i^{\text {th }}$ layer respectively, $i=1, \cdots, N$. We convert to spherical coordinates $\operatorname{Or} \theta \phi$ as depicted in Figure ??, where we have adopted the notation of (Spiegel, 1967).

Consider the position of rest $P(r, \theta, \phi)$ of a vibrating particle in the $i^{\text {th }}$ layer where $a_{i} \leq r \leq$ $a_{i-1}$. Let $\hat{\boldsymbol{r}}$ be the unit vector in the direction of increasing $r$. Hence the position vector of the point $P(r, \theta, \phi)$ is $\boldsymbol{r}=r \hat{\boldsymbol{r}}$. Consider the usual unit vectors $\hat{\boldsymbol{\phi}}=\frac{\partial \boldsymbol{r}}{\partial \phi} /\left|\frac{\partial \boldsymbol{r}}{\partial \phi}\right|$ (in the direction of increasing $\phi$ ) and $\hat{\boldsymbol{\theta}}=\frac{\partial \boldsymbol{r}}{\partial \theta} /\left|\frac{\partial \boldsymbol{r}}{\partial \theta}\right|$ (in the direction of increasing $\theta$ ). Let $\boldsymbol{w}_{i}+\boldsymbol{u}_{i}+\boldsymbol{v}_{i}$ (where $\boldsymbol{w}_{i}=w_{i} \hat{\boldsymbol{r}}, \boldsymbol{u}_{i}=u_{i} \hat{\boldsymbol{\theta}}$ and $\left.\boldsymbol{v}_{i}=v_{i} \hat{\boldsymbol{\phi}}\right)$ represent the displacement from the position of rest of the vibrating particle in the $i^{\text {th }}$ layer. For the sake of simplicity, we suppress subscripts $i$ when no confusion is expected. The position vector of the vibrating particle is thus

$$
\mathbf{R}=(r+w) \hat{\boldsymbol{r}}+u \hat{\boldsymbol{\theta}}+v \hat{\boldsymbol{\phi}} .
$$

Now consider an inertial coordinate system $O X Y Z$ with origin $O$, where initially the $X, Y, Z$-axes correspond to the $x, y, z$-axes respectively. Let the spherical body (the $\operatorname{Or} \theta \phi \equiv$ $O x y z$ system) rotate about the $z$-axis with respect to inertial space $O X Y Z$ with a small constant angular rate. If $\hat{k}$ is the unit vector in the direction of increasing $z$, then let the angular velocity $\Omega$ of the body be

$$
\Omega=\varepsilon \Omega \hat{\boldsymbol{k}}=\varepsilon \Omega(\hat{\boldsymbol{r}} \cos \theta-\hat{\boldsymbol{\theta}} \sin \theta),
$$




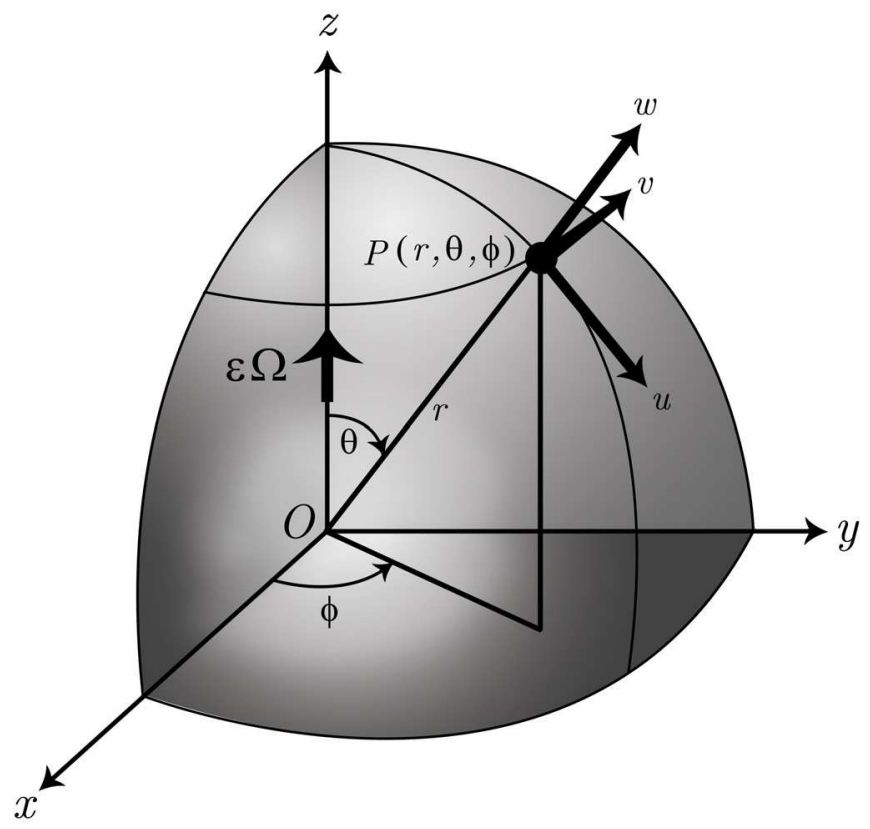

Fig. 1. The spherical coordinate system $\operatorname{Or} \theta \phi$ and its relation to the reference frame $O x y z$ that is rotating slowly about the $z$-axis at rate $\varepsilon \Omega$, showing a particle in the $i^{\text {th }}$ layer with position of rest $P(r, \theta, \phi)$ and displacement from the position of rest $u$ in the direction of increasing angle $\theta, v$ in the direction of increasing angle $\phi$ and $w$ in the radial direction.

where the dimensionless parameter $\varepsilon$ is a measure of smallness. By the word "smallness" of the angular rate of rotation $\varepsilon \Omega$ we mean (as mentioned above) that this rate is substantially smaller than the lowest eigenvalue of the system.

\section{Gyroscopic effects in distributed bodies}

The mathematical formulation given below (in spherical coordinates) is presented within the framework of the linearised, three-dimensional theory of elasticity (Redwood, 1960). With Lagrange's equations (Spiegel, 1967) in mind, we formulate expressions for the (approximate) kinetic and potential energies of the system of concentric spherical bodies. The absolute linear velocity of the vibrating particle is

$$
\begin{gathered}
\boldsymbol{V}=\frac{d \boldsymbol{R}}{d t}+\boldsymbol{\Omega} \times \boldsymbol{R}= \\
(\dot{w}-\varepsilon \Omega v \sin \theta) \hat{\boldsymbol{r}}+ \\
(\dot{u}-\varepsilon \Omega v \cos \theta) \hat{\boldsymbol{\theta}}+ \\
(\dot{v}+\varepsilon \Omega(u \cos \theta+(r+w) \sin \theta)) \hat{\boldsymbol{\phi}} .
\end{gathered}
$$


Neglecting terms of $O\left(\varepsilon^{2}\right)$, the approximate kinetic energy of the system of concentric spherical bodies is given by

$$
\begin{gathered}
K=\frac{1}{2} \sum_{i=1}^{N} \rho_{i} \int_{0}^{2 \pi} \int_{0}^{\pi} \int_{a_{i}}^{a_{i-1}}\left\{\left(\dot{u}_{i}^{2}+\dot{v}_{i}^{2}+\dot{w}_{i}^{2}\right)+2 \varepsilon \Omega\left[\left(u_{i} \dot{v}_{i}-\dot{u}_{i} v_{i}\right) \cos \theta+\right.\right. \\
\left.\left.\left(\dot{v}_{i}\left(r+w_{i}\right)-v_{i} \dot{w}_{i}\right) \sin \theta\right]\right\} r^{2} \sin \theta d r d \theta d \phi .
\end{gathered}
$$

The potential energy of the system of concentric spheres is

$$
\begin{gathered}
P=\frac{1}{2} \sum_{i=1}^{N} \int_{0}^{2 \pi} \int_{0}^{\pi} \int_{a_{i}}^{a_{i-1}}\left\{\sigma_{i, r r} \epsilon_{i, r r}+\sigma_{i, \theta \theta} \epsilon_{i, \theta \theta}+\sigma_{i, \phi \phi} \epsilon_{i, \phi \phi}+\right. \\
\left.\sigma_{i, r \theta} \epsilon_{i, r \theta}+\sigma_{i, \theta \phi} \epsilon_{i, \theta \phi}+\sigma_{i, r \phi} \epsilon_{i, r \phi}\right\} r^{2} \sin \theta d r d \theta d \phi
\end{gathered}
$$

where we use " $\rho$ " for mass density, " $\sigma$ " for stress and " $\epsilon$ " for strain. We indicate Lamé's constants (from the theory of elasticity) by $\lambda_{i}$ and $\mu_{i}$ for the $i^{\text {th }}$ layer. In a spherical coordinate system, with the subscript $i$ suppressed for the sake of brevity (i.e. we write $\sigma_{r r}$ for $\sigma_{i, r r}$ etc.), a standard reference such as (Redwood, 1960) yields stresses

$$
\begin{gathered}
\sigma_{r r}=\lambda\left(\epsilon_{r r}+\epsilon_{\theta \theta}+\epsilon_{\phi \phi}\right)+2 \mu \epsilon_{r r} ; \quad \sigma_{\theta \theta}=\lambda\left(\epsilon_{r r}+\epsilon_{\theta \theta}+\epsilon_{\phi \phi}\right)+2 \mu \epsilon_{\theta \theta} ; \\
\sigma_{\phi \phi}=\lambda\left(\epsilon_{r r}+\epsilon_{\theta \theta}+\epsilon_{\phi \phi}\right)+2 \mu \epsilon_{\phi \phi} ; \\
\sigma_{r \theta}=2 \mu \epsilon_{r \theta} ; \quad \sigma_{\theta \phi}=2 \mu \epsilon_{\theta \phi} ; \quad \sigma_{r \phi}=2 \mu \epsilon_{r \phi} ;
\end{gathered}
$$

and strains

$$
\begin{gathered}
\epsilon_{r r}=\frac{\partial w}{\partial r} ; \quad \epsilon_{\theta \theta}=\frac{1}{r}\left(\frac{\partial u}{\partial \theta}+w\right) ; \quad \epsilon_{\phi \phi}=\frac{1}{r}\left(u \cot \theta+\frac{1}{\sin \theta} \frac{\partial v}{\partial \phi}+w\right) ; \\
\epsilon_{r \theta}=\frac{\partial u}{\partial r}+\frac{1}{r}\left(\frac{\partial w}{\partial \theta}-u\right) ; \quad \epsilon_{\theta \phi}=\frac{1}{r}\left(\frac{1}{\sin \theta} \frac{\partial u}{\partial \phi}+\frac{\partial v}{\partial \theta}-v \cot \theta\right) ; \\
\epsilon_{r \phi}=\frac{\partial v}{\partial r}+\frac{1}{r}\left(\frac{1}{\sin \theta} \frac{\partial w}{\partial \phi}-v\right) .
\end{gathered}
$$

Assume that we can express the magnitude of the displacements $u_{i}, v_{i}$ and $w_{i}$ of the vibrating particle $P$ in the $i^{t h}$ body as follows:

$$
\begin{aligned}
u_{i}(r, \theta, \phi, t) & =U_{i}(r, \theta)[C(t) \cos m \phi+S(t) \sin m \phi], \\
v_{i}(r, \theta, \phi, t) & =V_{i}(r, \theta)[C(t) \sin m \phi-S(t) \cos m \phi], \\
w_{i}(r, \theta, \phi, t) & =W_{i}(r, \theta)[C(t) \cos m \phi+S(t) \sin m \phi],
\end{aligned}
$$

where the nature of the functions $C(t)$ and $S(t)$ is still to be determined, $U_{i}(r, \theta), V_{i}(r, \theta)$ and $W_{i}(r, \theta)$ are unknown eigenfunctions of the system and $m$ is the circumferential wave number. Substituting Equations (9) into Equations (8), (7), (6) and (5) involves a long algebraic calculation. A computer algebra system is handy for checking the calculation that yields:

$$
\left.K=\pi\left[I_{0}\left(\dot{C}^{2}+\dot{S}^{2}\right)+2 \Omega I_{1}(\dot{C} S-C \dot{S})\right)\right]
$$


and

$$
P=\pi I_{2}\left(C^{2}+S^{2}\right)
$$

Here

$$
\begin{aligned}
& I_{0}=\frac{1}{2} \sum_{i=1}^{N} \rho_{i} \int_{0}^{\pi} \int_{a_{i}}^{a_{i-1}}\left(U_{i}^{2}+V_{i}^{2}+W_{i}^{2}\right) r^{2} \sin \theta d r d \theta, \\
& I_{1}=\sum_{i=1}^{N} \rho_{i} \int_{0}^{\pi} \int_{a_{i}}^{a_{i-1}}\left(U_{i} \cos \theta+W_{i} \sin \theta\right) V_{i} r^{2} \sin \theta d r d \theta \\
& I_{2}=\sum_{i=1}^{N} \rho_{i} \int_{0}^{\pi} \int_{a_{i}}^{a_{i-1}} \\
& \left\{2 \mu_{i}\left[\begin{array}{c}
\left(m \csc \theta U_{i}+\cot \theta V_{i}-\frac{\partial V_{i}}{\partial \theta}\right)^{2}+ \\
\left.\left(\frac{\partial W_{i}}{\partial \theta}+r \frac{\partial U_{i}}{\partial r}-U_{i}\right)^{2}+\left(V_{i}+m \csc \theta W_{i}\right)-r \frac{\partial V_{i}}{\partial r}\right)^{2}
\end{array}\right]+\right. \\
& \left(\cot \theta U_{i}+m \csc \theta V_{i}+W_{i}\right) \times \\
& {\left[\left(\lambda_{i}+2 \mu_{i}\right)\left[\begin{array}{c}
\cot \theta U_{i}+ \\
m \csc \theta V_{i}+2\left(\lambda_{i}+\mu_{i}\right) W
\end{array}\right]+\lambda_{i}\left(\frac{\partial U_{i}}{\partial \theta}+r \frac{\partial W_{i}}{\partial r}\right)\right]+} \\
& \left(W_{i}+\frac{\partial U_{i}}{\partial \theta}\right)\left(\begin{array}{c}
\lambda_{i}\left(\cot \theta U_{i}+m \csc \theta V_{i}\right)+ \\
2\left(\lambda_{i}+\mu_{i}\right) W_{i}+\left(\lambda_{i}+2 \mu_{i}\right) \frac{\partial U_{i}}{\partial \theta}+r \lambda_{i} \frac{\partial W_{i}}{\partial \theta}
\end{array}\right)+ \\
& \left.r \frac{\partial W_{i}}{\partial r}\left[\lambda_{i}\left(\begin{array}{c}
\cot \theta U_{i}+m \csc \theta V_{i}+ \\
2 W_{i}+\frac{\partial U_{i}}{\partial \theta}
\end{array}\right)+r\left(\lambda_{i}+2 \mu_{i}\right) \frac{\partial W_{i}}{\partial r}\right]\right\} \sin \theta d r d \theta .
\end{aligned}
$$

Because $K=K(C, S, \dot{C}, \dot{S})$ and $P=P(C, S)$, the Lagrangian

$$
L(C, S, \dot{C}, \dot{S})=K-P
$$

yields two equations of motion from Lagrange's equations (Goldstein, et al.):

$$
\begin{aligned}
\frac{d}{d t} \frac{\partial L}{\partial \dot{C}}-\frac{\partial L}{\partial C} & =-\frac{\partial \mathcal{F}}{\partial \dot{C}} \\
\frac{d}{d t} \frac{\partial L}{\partial \dot{S}}-\frac{\partial L}{\partial S} & =-\frac{\partial \mathcal{F}}{\partial \dot{S}}
\end{aligned}
$$

where

$$
\mathcal{F}=\frac{1}{2}\left(c \dot{C}^{2}+s \dot{S}^{2}\right)
$$

is Rayleigh's dissipation function, $c$ and $s$ are viscous damping constants. We assume isotropic damping, that is, say, $c=s=\pi D$. We further assume that for the media we will encounter, the "damping factor"

$$
\varepsilon \delta=\frac{D}{2 I_{0}}
$$


is substantially smaller than the lowest eigenvalue of the vibrating system. Computation of Equations (16) yields a coupled system of second-order linear ordinary differential equations (ODE):

$$
\begin{aligned}
\ddot{C}+2 \eta \varepsilon \Omega \dot{S}+\omega^{2} C+2 \varepsilon \delta \dot{C} & =0, \\
\ddot{S}-2 \eta \varepsilon \Omega \dot{C}+\omega^{2} S+2 \varepsilon \delta \dot{S} & =0,
\end{aligned}
$$

where

$$
-1 \leq \eta=\frac{I_{1}}{I_{0}} \leq 1
$$

and

$$
\omega^{2}=\frac{I_{2}}{I_{0}}
$$

We now show that $\eta$ in Equation (20) is Bryan's factor as given by Equation (1) and that $\omega$ in Equation (21) is an eigenvalue for the vibrating system.

In order to interpret what the system of ODE (19) represents, combine the two equations by considering the complex function $\mathrm{Z}=\mathrm{C}+i S$ to obtain the single equation

$$
\ddot{Z}+2 \varepsilon(\delta-i \eta \Omega) \dot{Z}+\omega^{2} Z=0 .
$$

where, as is usual, $i^{2}=-1$. Writing $\mathrm{Z}$ in polar form

$$
Z(t)=Y(t) e^{i \beta(t)}
$$

and assuming that $\beta(t)$ has the linear form

$$
\beta(t)=a t
$$

while $Y(t)$ decays according to

$$
Y(t)=X(t) e^{-b t}
$$

then

$$
Z=X(t) e^{(i a-b) t}
$$

and substituting into Equation (22), we obtain the ODE

$$
\begin{gathered}
\ddot{X}+2[(i a-b)+\varepsilon(\delta-i \eta \Omega)] \dot{X}+ \\
{\left[2 \varepsilon(\delta-i \eta \Omega)(i a-b)+(i a-b)^{2}+\omega^{2}\right] X=0 .}
\end{gathered}
$$

If we choose $a=\eta \varepsilon \Omega$ and $b=\varepsilon \delta$, then the coefficient of $\dot{X}$ vanishes in Equation (27) and we obtain the ODE

$$
\ddot{X}+v^{2} X=0
$$

where

$$
v^{2}=\omega^{2}-\varepsilon^{2}(\delta-i \eta \Omega)^{2}
$$

Neglecting $O\left(\varepsilon^{2}\right)$, we obtain

$$
v \approx \omega
$$

Consequently Equation (28) approximates the equation of motion of a harmonic oscillator. Equations (19) can now be viewed in the form

$$
Z(t)=\left[e^{-\delta t} X(t)\right] e^{i \eta \varepsilon \Omega t}
$$


These equations represent a "vector" in the complex plane with its size varying like a damped harmonic oscillator and its position varying at a rate $\eta \varepsilon \Omega$ (in the rotating reference frame Oxyz). Thus, according to Equation (1), $\eta$ is Bryan's factor for the system. Consequently, neither Bryan's effect nor the value of Bryan's factor $\eta$, depends on the inclusion of light isotropic viscous damping in the model and we will thus neglect damping in the sequel. The rotation of the vibrating pattern is in the direction of rotation of the system if $\eta>0$ and in the opposite direction if $\eta<0$. Equations (28) and (30) show that $\omega$ is an eigenvalue of the vibrating system.

Taking Equation (30) into account, Equation (28) has a general solution of the form

$$
X(t)=A \cos (\omega t+\gamma)
$$

where the amplitude $A$ and phase angle $\gamma$ are arbitrary constants. Consequently, neglecting damping, from Equation (31) we obtain

$$
C+i S=A \cos (\omega t+\gamma) e^{i \eta \varepsilon \Omega t} .
$$

Equating real and imaginary parts we have a good approximation

$$
\begin{aligned}
& C(t)=A \cos \eta \varepsilon \Omega t \cos (\omega t+\gamma), \\
& S(t)=A \sin \eta \varepsilon \Omega t \cos (\omega t+\gamma)
\end{aligned}
$$

and so initially, that is, when $t \approx 0$, we find that

$$
\begin{aligned}
& C(t) \approx A \cos (\omega t+\gamma), \\
& S(t) \approx 0 .
\end{aligned}
$$

\section{Eigenfunctions}

In order to determine the nature of Bryan's constant $\eta$ (Equation (20)) and the square of the corresponding eigenvalue $\omega$ (Equation (21)), we neglected $O\left(\varepsilon^{2}\right)$. We are going to demonstrate that it is also feasible to neglect the small rotation rate $\varepsilon \Omega$ when we derive expressions for the eigenfunctions $U_{i}(r, \theta), V_{i}(r, \theta)$ and $W_{i}(r, \theta), i=1, \cdots, N$, as given in Equations (9). Indeed, assume that we have a system of partial differential equations (PDE) involving the eigenfunctions $U_{i}(r, \theta), V_{i}(r, \theta)$ and $W_{i}(r, \theta)$. Using the small parameter $\varepsilon$ and a perturbation method Nayfeh (1973), assume that we have an asymptotic expansion

$$
U_{i}(r, \theta)=U_{i, 0}(r, \theta)+\varepsilon U_{i, 1}(r, \theta)+\varepsilon^{2} U_{i, 2}(r, \theta)+\cdots,
$$

where the $U_{i, j}(r, \theta), j=0,1,2, \cdots$ are independent of $\varepsilon$ and $U_{i, 0}(r, \theta)$ is that part of the solution when $\varepsilon=0$. A similar argument is valid for the eigenfunctions $V_{i}(r, \theta)$ and $W_{i}(r, \theta)$. Substituting these three expansions into Equations (12) and (13) and again neglecting all term of $O\left(\varepsilon^{2}\right)$, the formula for $\eta$ given in Equation (20) yields

$$
\eta=\frac{2 \sum_{i=1}^{N} \rho_{i} \int_{0}^{\pi} \int_{a_{i}}^{a_{i-1}}\left[\left(U_{i, 0} \cos \theta+W_{i, 0} \sin \theta\right) V_{i, 0}+O(\varepsilon)\right] r^{2} \sin \theta d r d \theta}{\sum_{i=1}^{N} \rho_{i} \int_{0}^{\pi} \int_{a_{i}}^{a_{i-1}}\left[U_{i, 0}^{2}+V_{i, 0}^{2}+W_{i, 0}^{2}+O(\varepsilon)\right] r^{2} \sin \theta d r d \theta} .
$$


Now write Bryan's factor $\eta$ as an asymptotic expansion

$$
\eta=\eta_{0}+\varepsilon \eta_{1}+\varepsilon^{2} \eta_{2}+\cdots
$$

where the $\eta_{j}, j=0,1,2, \cdots$ are independent of $\varepsilon$ and $\eta_{0}$ is given by Equation (38) when $\varepsilon=0$, that is

$$
\eta_{0}=\frac{2 \sum_{i=1}^{N} \rho_{i} \int_{0}^{\pi} \int_{a_{i}}^{a_{i-1}}\left[\left(U_{i, 0} \cos \theta+W_{i, 0} \sin \theta\right) V_{i, 0}\right] r^{2} \sin \theta d r d \theta}{\sum_{i=1}^{N} \rho_{i} \int_{0}^{\pi} \int_{a_{i}}^{a_{i-1}}\left[U_{i, 0}^{2}+V_{i, 0}^{2}+W_{i, 0}^{2}\right] r^{2} \sin \theta d r d \theta} .
$$

Hence, because we neglect $O\left(\varepsilon^{2}\right)$, Equation (39) yields

$$
\varepsilon \eta=\varepsilon \eta_{0}+O\left(\varepsilon^{2}\right) \approx \varepsilon \eta_{0}
$$

and so

$$
\eta \approx \eta_{0}
$$

A similar result is valid for the square of the eigenvalue $\omega$ determined by Equation (21). Consequently, because only the eigenfunctions of a non-rotating body need be considered for the purposes of numerically calculating $\eta$, in the sequel we will use the notation $u_{i}$ for $u_{i, 0}$, $U_{i}$ for $U_{i, 0}$, etc. Indeed, using (Redwood, 1960) and our notation for stresses, the equations of motion of an isotropic solid, nonrotating vibrating body in spherical coordinates are

$$
\begin{gathered}
\rho \frac{\partial^{2} u}{\partial t^{2}}=\frac{\partial \sigma_{r r}}{\partial r}+\frac{1}{r} \frac{\partial \sigma_{r \theta}}{\partial \theta}+\frac{1}{r \sin \theta} \frac{\partial \sigma_{r \phi}}{\partial \phi}+\frac{2 \sigma_{r r}-\sigma_{\theta \theta}-\sigma_{\phi \phi}+\cot \theta \sigma_{r \theta}}{r}, \\
\rho \frac{\partial^{2} v}{\partial t^{2}}=\frac{\partial \sigma_{r \theta}}{\partial r}+\frac{1}{r} \frac{\partial \sigma_{\theta \theta}}{\partial \theta}+\frac{1}{r \sin \theta} \frac{\partial \sigma_{\theta \phi}}{\partial \phi}+\frac{3 \sigma_{r \theta}+\cot \theta\left(\sigma_{\theta \theta}-\sigma_{\phi \phi}\right)}{r}, \\
\rho \frac{\partial^{2} w}{\partial t^{2}}=\frac{\partial \sigma_{r \phi}}{\partial r}+\frac{1}{r} \frac{\partial \sigma_{\theta \phi}}{\partial \theta}+\frac{1}{r \sin \theta} \frac{\partial \sigma_{\phi \phi}}{\partial \phi}+\frac{3 \sigma_{r \phi}+2 \cot \theta \sigma_{\theta \phi}}{r} .
\end{gathered}
$$

The stresses are given by Equation (7). The coupled system of second-order PDE given by Equations (43) for the three displacement components $u, v$ and $w$ can be uncoupled directly, but this leads to a sixth-order PDE. Hence, in a manner similar to that explained in (Eringen \& Suhubi, 1975), we express the displacement components of Equations (43) in terms of derivatives of potentials $\Phi=\Phi(r, \theta, \phi), \chi=\chi(r, \theta, \phi)$ and $\Psi=\Psi(r, \theta, \phi)$ as follows:

$$
\begin{aligned}
u & =\left\{\frac{1}{r} \frac{\partial}{\partial \theta}\left[\Phi+\frac{\partial(r \chi)}{\partial r}\right]+\frac{1}{a \sin \theta} \frac{\partial \Psi}{\partial \phi}\right\} \cos (\omega t+\gamma), \\
v & =\left\{\frac{1}{r \sin \theta} \frac{\partial}{\partial \phi}\left[\Phi+\frac{\partial(r \chi)}{\partial r}\right]-\frac{1}{a} \frac{\partial \Psi}{\partial \theta}\right\} \cos (\omega t+\gamma), \\
w & =\left\{\frac{\partial \Phi}{\partial r}+\frac{\partial^{2}(r \chi)}{\partial r^{2}}+r \nabla^{2} \chi\right\} \cos (\omega t+\gamma),
\end{aligned}
$$

where $\nabla^{2}$ the Laplace operator in spherical coordinates, $\omega$ is the eigenvalue mentioned above, $\gamma$ is some phase angle (see Equation (32)) and $a$ is a nonzero constant with the dimension of length. When Equations (44) are substituted into Equations (43) and the resulting equations of motion are uncoupled, it is found that each potential $\Phi, \chi$ and $\Psi$ satisfies the Helmholtz equations

$$
\nabla^{2} \Phi+k_{1}^{2} \Phi=0, \quad \nabla^{2} \chi+k_{2}^{2} \chi=0, \quad \nabla^{2} \Psi+k_{2}^{2} \Psi=0
$$


with wave numbers

$$
\begin{aligned}
& k_{1}=k_{1}(\omega)=\omega \sqrt{\rho /(\lambda+2 \mu),} \\
& k_{2}=k_{2}(\omega)=\omega \sqrt{\rho / \mu} .
\end{aligned}
$$

In order to obtain initial (at $t \approx 0$ ) agreement with our choice of Equations (9), the appropriate solutions to Equations (45) are

$$
\begin{gathered}
\Phi_{m, n}(r, \theta, \phi)= \\
{\left[B_{1} j_{n}\left(k_{1} r\right)+B_{2} y_{n}\left(k_{1} r\right)\right] P_{n}^{m}(\cos \theta) \cos (m \phi),} \\
\chi_{m, n}(r, \theta, \phi)= \\
{\left[B_{3} j_{n}\left(k_{2} r\right)+B_{4} y_{n}\left(k_{2} r\right)\right] P_{n}^{m}(\cos \theta) \cos (m \phi),} \\
\Psi_{m, n}(r, \theta, \phi)= \\
{\left[B_{5} j_{n}\left(k_{2} r\right)+B_{6} y_{n}\left(k_{2} r\right)\right] P_{n}^{m}(\cos \theta) \sin (m \phi),}
\end{gathered}
$$

where, as usual, $j_{n}(k r)=\sqrt{\frac{\pi}{2 k r}} J_{n+1 / 2}(k r)$ and $y_{n}(k r)=\sqrt{\frac{\pi}{2 k r}} Y_{n+1 / 2}(k r)$ represent the spherical Bessel and Neumann functions respectively where $J_{n+1 / 2}$ and $Y_{n+1 / 2}$ represent the Bessel and Neumann functions respectively, while $P_{n}^{m}$ is the associated Legendre polynomial. The symbols $B_{1}, B_{2}, \cdots, B_{6}$ are arbitrary constants (if the body contains the centre $O$, then the constants $B_{2}=B_{4}=B_{6}=0$ ). By substituting Equations (47) into Equations (44) we obtain the nature of the eigenfunctions $U(r, \theta), V(r, \theta)$ and $W(r, \theta)$ as given in Equations (9).

The motion of a compressible, inviscid acoustic medium is represented by the following wave equation:

$$
\nabla^{2} p=\frac{\rho}{\kappa} \frac{\partial^{2} p}{\partial t^{2}}
$$

where $\kappa$ is the bulk modulus and $\rho$ the mass density of the acoustic medium. With the wave number

$$
k_{3}=k_{3}(\omega)=\omega \sqrt{\rho / \kappa},
$$

an appropriate solution to PDE (48) is

$$
\begin{aligned}
p_{m, n}(r, \theta, \phi, t) & = \\
& \left\{\left[B_{7} j_{n}\left(k_{3} r\right)+B_{8} y_{n}\left(k_{3} r\right)\right] P_{n}^{m}(\cos \theta) \cos (m \phi)\right\} \cos (\omega t+\gamma),
\end{aligned}
$$

where $p=p_{m, n}(r, \theta, \phi, t)$ is the pressure in the acoustic medium and $B_{7}, B_{8}$ are arbitrary constants. Particle displacement of the acoustic medium in the radial direction is

$$
w=\frac{1}{\rho \omega^{2}} \frac{\partial p}{\partial r} .
$$

In the sequel, keep in mind that for $j=1,2,3$ and layers $i=1, \cdots, N$, we will write $k_{i, j}$ for $k_{j}$ in layer $i$, etc.

\section{Boundary and continuity conditions}

Observing Equations (44) it is possible to distinguish between spheroidal and torsional modes. For the spheroidal mode we assume that $\Psi_{i}=0$. In this case the stress components of the 
solids are

$$
\begin{aligned}
\sigma_{i, r r} & =\left[2 \mu_{i} \frac{\partial^{2} \Phi_{i}}{\partial r^{2}}-\lambda_{i} k_{i, 1}^{2} \Phi_{i}\right]+2 \mu_{i}\left[\frac{\partial^{3}\left(r \chi_{i}\right)}{\partial r^{3}}+k_{i, 2}^{2} \frac{\partial\left(r \chi_{i}\right)}{\partial r}\right], \\
\sigma_{i, r \theta} & =\frac{2 \mu_{i}}{r} \frac{\partial}{\partial \theta}\left\{\left(\frac{\partial \Phi_{i}}{\partial r}-\frac{\Phi_{i}}{r}\right)+\left[r \frac{\partial^{2} \chi_{i}}{\partial r^{2}}+\frac{\partial \chi_{i}}{\partial r}+\left(\frac{r k_{i, 2}^{2}}{2}-\frac{1}{r}\right) \chi_{i}\right]\right\}, \\
\sigma_{i, r \phi} & =\frac{2 \mu_{i}}{r \sin \theta} \frac{\partial}{\partial \phi}\left\{\left(\frac{\partial \Phi_{i}}{\partial r}-\frac{\Phi_{i}}{r}\right)+\left[r \frac{\partial^{2} \chi_{i}}{\partial r^{2}}+\frac{\partial \chi_{i}}{\partial r}+\left(\frac{r k_{i, 2}^{2}}{2}-\frac{1}{r}\right) \chi_{i}\right]\right\},
\end{aligned}
$$

where $i=1,2,3,4,6$ denotes a solid layer in the spherical structure. Indeed, here we assume $i=1$ for the outer solid layer ("crust"), $i=2$ for the next inner layer ("upper mantle-1"), $i=3$ for the "upper mantle-2", $i=4$ for the "lower mantle" and $i=6$ for the "inner solid core". We do not use $i=5$ in the list of the solids because this describes the "liquid outer core".

For the investigation of the torsional modes we assume that $\Phi_{i}=0$ and $\chi_{i}=0$ and hence the corresponding stress components are

$$
\begin{aligned}
\sigma_{i, r \theta} & =\frac{\mu_{i}}{a \sin \theta} \frac{\partial}{\partial \phi}\left(\frac{\partial \Psi_{i}}{\partial r}-\frac{\Psi_{i}}{r}\right), \\
\sigma_{i, r \phi} & =-\frac{\mu_{i}}{a} \frac{\partial}{\partial \theta}\left(\frac{\partial \Psi_{i}}{\partial r}-\frac{\Psi_{i}}{r}\right) .
\end{aligned}
$$

Let us model a spherical "planet" with solid "inner core", four solid layers and one liquid layer. Assume that the outer radius of the planet is $r=a$ and that the boundary between the "crust" and the "upper mantle-1" is located at radius $r=a_{1}$. Furthermore, assume that the boundary between the "upper mantle-1" and the "upper mantle-2" is situated at radius $r=a_{2}$, while at $r=a_{3}$ we find the boundary between the "upper mantle-2" and the "lower mantle". All these layers are considered as isotropic solid bodies. Assume that the boundary between the "lower mantle" and the "liquid outer core" is situated at radius $r=a_{4}$ and finally, radius $r=a_{5}$ is the boundary between the "liquid outer core" and the solid "inner core".

The elastic potentials and pressure that describe the spheroidal modes of the planet are solutions given by Equations (47a), (47b) and (50):

$$
\begin{aligned}
\Phi_{i}(r, \theta, \phi) & =\left[A_{4 i-3} j_{n}\left(k_{i, 1} r\right)+A_{4 i-2} y_{n}\left(k_{i, 1} r\right)\right] P_{n}^{m}(\cos \theta) \cos m \phi, \\
\chi_{i}(r, \theta, \phi) & =\left[A_{4 i-1} j_{n}\left(k_{i, 2} r\right)+A_{4 i} y_{n}\left(k_{i, 2} r\right)\right] P_{n}^{m}(\cos \theta) \cos m \phi, \\
p_{5}(r, \theta, \phi) & =\left[A_{17} j_{n}\left(k_{5,3} r\right)+A_{18} y_{n}\left(k_{5,3} r\right)\right] P_{n}^{m}(\cos \theta) \cos m \phi, \\
\Phi_{6}(r, \theta, \phi) & =\left[A_{19} j_{n}\left(k_{6,1} r\right)\right] P_{n}^{m}(\cos \theta) \cos m \phi, \\
\chi_{6}(r, \theta, \phi) & =\left[A_{20} j_{n}\left(k_{6,2} r\right)\right] P_{n}^{m}(\cos \theta) \cos m \phi,
\end{aligned}
$$

where the symbols " $A$ " with various subscripts represent arbitrary constants that will be determined from the boundary and continuity conditions described below and $i=1,2,3,4$. The "upper core" of the planet does not have a torsional mode and is considered to be an inviscid ideal fluid. Despite the fact that the "inner core" is solid and hence that it could support the torsional mode, we do not consider it when we analyse the torsional mode because the shear stress in the "inner core" cannot be transformed into the outer layers through the shear free liquid "outer core". The elastic potential describing the torsional modes of the planet is obtained from Equation (47c):

$$
\Psi_{i}(r, \theta, \phi)=\left[B_{2 i-1} j_{n}\left(k_{i, 2} r\right)+A_{2 i} y_{n}\left(k_{i, 2} r\right)\right] P_{n}^{m}(\cos \theta) \cos m \phi,
$$

where $i=1,2,3,4$. 
The boundary and continuity conditions below express the balance between the radial stresses and pressure and equality of the tangential stresses in the solid layers. They also express the equality of the radial and tangential components between the solid layers (correspondingly, only radial components between the solids and fluid layer). The outer layer is assumed to be free from radial and tangential stress components. Using these assumptions together with Equations (52) we obtain the following boundary and continuity conditions for the investigation of the spheroidal modes:

At $r=a_{0}=a$

$$
\begin{gathered}
\left.\left\{\left[2 \mu_{1} \frac{\partial^{2} \Phi_{1}}{\partial r^{2}}-\lambda_{1} k_{1,1}^{2} \Phi_{1}\right]+2 \mu_{1}\left[\frac{\partial^{3}\left(r \chi_{1}\right)}{\partial r^{3}}+k_{1,2}^{2} \frac{\partial\left(r \chi_{1}\right)}{\partial r}\right]\right\}\right|_{r=a}=0 \\
\left.\frac{2 \mu_{1}}{a}\left\{\left(\frac{\partial \Phi_{1}}{\partial r}-\frac{\Phi_{1}}{r}\right)+\left[r \frac{\partial^{2} \chi_{1}}{\partial r^{2}}+\frac{\partial \chi_{1}}{\partial r}+\left(\frac{r k_{1,2}^{2}}{2}-\frac{1}{r}\right) \chi_{1}\right]\right\}\right|_{r=a}=0
\end{gathered}
$$

At $r=a_{1}$

$$
\begin{gathered}
\left.\left\{\left[2 \mu_{1} \frac{\partial^{2} \Phi_{1}}{\partial r^{2}}-\lambda_{1} k_{1,1}^{2} \Phi_{1}\right]+2 \mu_{1}\left[\frac{\partial^{3}\left(r \chi_{1}\right)}{\partial r^{3}}+k_{1,2}^{2} \frac{\partial\left(r \chi_{1}\right)}{\partial r}\right]\right\}\right|_{r=a_{1}}= \\
\left.\left\{\left[2 \mu_{2} \frac{\partial^{2} \Phi_{2}}{\partial r^{2}}-\lambda_{2} k_{2,1}^{2} \Phi_{2}\right]+2 \mu_{2}\left[\frac{\partial^{3}\left(r \chi_{2}\right)}{\partial r^{3}}+k_{2,2}^{2} \frac{\partial\left(r \chi_{2}\right)}{\partial r}\right]\right\}\right|_{r=a_{1}}{ }^{\prime} \\
\left.\frac{2 \mu_{1}}{a_{1}}\left\{\left(\frac{\partial \Phi_{1}}{\partial r}-\frac{\Phi_{1}}{r}\right)+\left[r \frac{\partial^{2} \chi_{1}}{\partial r^{2}}+\frac{\partial \chi_{1}}{\partial r}+\left(\frac{r k_{1,2}^{2}}{2}-\frac{1}{r}\right) \chi_{1}\right]\right\}\right|_{r=a_{1}}= \\
\left.\frac{2 \mu_{2}}{a_{1}}\left\{\left(\frac{\partial \Phi_{2}}{\partial r}-\frac{\Phi_{2}}{r}\right)+\left[r \frac{\partial^{2} \chi_{2}}{\partial r^{2}}+\frac{\partial \chi_{2}}{\partial r}+\left(\frac{r k_{2,2}^{2}}{2}-\frac{1}{r}\right) \chi_{2}\right]\right\}\right|_{r=a_{1}}, \\
\left.\left\{\frac{\partial \Phi_{1}}{\partial r}+\left[\frac{\partial^{2}\left(r \chi_{1}\right)}{\partial r^{2}}+k_{1,2}^{2}\left(r \chi_{1}\right)\right]\right\}\right|_{r=a_{1}}= \\
\left.\left\{\frac{\partial \Phi_{2}}{\partial r}+\left[\frac{\partial^{2}\left(r \chi_{2}\right)}{\partial r^{2}}+k_{2,2}^{2}\left(r \chi_{2}\right)\right]\right\}\right|_{r=a_{1}}{ }^{\prime} \\
\left.\left\{\Phi_{1}+\frac{\partial\left(r \chi_{1}\right)}{\partial r}\right\}\right|_{r=a_{1}}=\left.\left\{\Phi_{2}+\frac{\partial\left(r \chi_{2}\right)}{\partial r}\right\}\right|_{r=a_{1}} .
\end{gathered}
$$

At $r=a_{2}$

$$
\begin{gathered}
\left.\left\{\left[2 \mu_{2} \frac{\partial^{2} \Phi_{2}}{\partial r^{2}}-\lambda_{2} k_{2,1}^{2} \Phi_{2}\right]+2 \mu_{2}\left[\frac{\partial^{3}\left(r \chi_{2}\right)}{\partial r^{3}}+k_{2,2}^{2} \frac{\partial\left(r \chi_{2}\right)}{\partial r}\right]\right\}\right|_{r=a_{2}}= \\
\left.\left\{\left[2 \mu_{3} \frac{\partial^{2} \Phi_{3}}{\partial r^{2}}-\lambda_{3} k_{3,1}^{2} \Phi_{3}\right]+2 \mu_{3}\left[\frac{\partial^{3}\left(r \chi_{3}\right)}{\partial r^{3}}+k_{3,2}^{2} \frac{\partial\left(r \chi_{3}\right)}{\partial r}\right]\right\}\right|_{r=a_{2}}{ }^{\prime} \\
\left.\frac{2 \mu_{2}}{a_{2}}\left\{\left(\frac{\partial \Phi_{2}}{\partial r}-\frac{\Phi_{2}}{r}\right)+\left[r \frac{\partial^{2} \chi_{2}}{\partial r^{2}}+\frac{\partial \chi_{2}}{\partial r}+\left(\frac{r k_{2,2}^{2}}{2}-\frac{1}{r}\right) \chi_{2}\right]\right\}\right|_{r=a_{2}}= \\
\left.\frac{2 \mu_{3}}{a_{2}}\left\{\left(\frac{\partial \Phi_{3}}{\partial r}-\frac{\Phi_{3}}{r}\right)+\left[r \frac{\partial^{2} \chi_{3}}{\partial r^{2}}+\frac{\partial \chi_{3}}{\partial r}+\left(\frac{r k_{3,2}^{2}}{2}-\frac{1}{r}\right) \chi_{3}\right]\right\}\right|_{r=a_{2}} \\
\left.\left\{\frac{\partial \Phi_{2}}{\partial r}+\left[\frac{\partial^{2}\left(r \chi_{2}\right)}{\partial r^{2}}+k_{2,2}^{2}\left(r \chi_{2}\right)\right]\right\}\right|_{r=a_{2}}= \\
\left.\left\{\frac{\partial \Phi_{3}}{\partial r}+\left[\frac{\partial^{2}\left(r \chi_{3}\right)}{\partial r^{2}}+k_{3,2}^{2}\left(r \chi_{3}\right)\right]\right\}\right|_{r=a_{2}},
\end{gathered}
$$




$$
\left.\left\{\Phi_{2}+\frac{\partial\left(r \chi_{2}\right)}{\partial r}\right\}\right|_{r=a_{2}}=\left.\left\{\Phi_{3}+\frac{\partial\left(r \chi_{3}\right)}{\partial r}\right\}\right|_{r=a_{2}} .
$$

At $r=a_{3}$

$$
\begin{gathered}
\left.\left\{\left[2 \mu_{3} \frac{\partial^{2} \Phi_{3}}{\partial r^{2}}-\lambda_{3} k_{3,1}^{2} \Phi_{3}\right]+2 \mu_{3}\left[\frac{\partial^{3}\left(r \chi_{3}\right)}{\partial r^{3}}+k_{3,2}^{2} \frac{\partial\left(r \chi_{3}\right)}{\partial r}\right]\right\}\right|_{r=a_{3}}= \\
\left.\left\{\left[2 \mu_{4} \frac{\partial^{2} \Phi_{4}}{\partial r^{2}}-\lambda_{4} k_{4,1}^{2} \Phi_{4}\right]+2 \mu_{4}\left[\frac{\partial^{3}\left(r \chi_{4}\right)}{\partial r^{3}}+k_{4,2}^{2} \frac{\partial\left(r \chi_{4}\right)}{\partial r}\right]\right\}\right|_{r=a_{3}}, \\
\left.\frac{2 \mu_{3}}{a_{3}}\left\{\left(\frac{\partial \Phi_{3}}{\partial r}-\frac{\Phi_{3}}{r}\right)+\left[r \frac{\partial^{2} \chi_{3}}{\partial r^{2}}+\frac{\partial \chi_{3}}{\partial r}+\left(\frac{r k_{3,2}^{2}}{2}-\frac{1}{r}\right) \chi_{3}\right]\right\}\right|_{r=a_{3}}= \\
\left.\frac{2 \mu_{4}}{a_{3}}\left\{\left(\frac{\partial \Phi_{4}}{\partial r}-\frac{\Phi_{4}}{r}\right)+\left[r \frac{\partial^{2} \chi_{4}}{\partial r^{2}}+\frac{\partial \chi_{4}}{\partial r}+\left(\frac{r k_{4,2}^{2}}{2}-\frac{1}{r}\right) \chi_{4}\right]\right\}\right|_{r=a_{3}}, \\
\left.\left\{\frac{\partial \Phi_{3}}{\partial r}+\left[\frac{\partial^{2}\left(r \chi_{3}\right)}{\partial r^{2}}+k_{3,2}^{2}\left(r \chi_{3}\right)\right]\right\}\right|_{r=a_{3}}= \\
\left.\left\{\frac{\partial \Phi_{4}}{\partial r}+\left[\frac{\partial^{2}\left(r \chi_{4}\right)}{\partial r^{2}}+k_{4,2}^{2}\left(r \chi_{4}\right)\right]\right\}\right|_{r=a_{3}}, \\
\left.\left\{\Phi_{3}+\frac{\partial\left(r \chi_{3}\right)}{\partial r}\right\}\right|_{r=a_{3}}=\left.\left\{\Phi_{4}+\frac{\partial\left(r \chi_{4}\right)}{\partial r}\right\}\right|_{r=a_{3}} .
\end{gathered}
$$

At $r=a_{4}$

$$
\begin{gathered}
\left.\left\{\left[2 \mu_{4} \frac{\partial^{2} \Phi_{4}}{\partial r^{2}}-\lambda_{4} k_{4,1}^{2} \Phi_{4}\right]+2 \mu_{4}\left[\frac{\partial^{3}\left(r \chi_{4}\right)}{\partial r^{3}}+k_{4,2}^{2} \frac{\partial\left(r \chi_{4}\right)}{\partial r}\right]\right\}\right|_{r=a_{4}}=-\left.p_{5}\right|_{r=a_{4}}, \\
\left.\frac{2 \mu_{4}}{a_{4}}\left\{\left(\frac{\partial \Phi_{4}}{\partial r}-\frac{\Phi_{4}}{r}\right)+\left[r \frac{\partial^{2} \chi_{4}}{\partial r^{2}}+\frac{\partial \chi_{4}}{\partial r}+\left(\frac{r k_{4,2}^{2}}{2}-\frac{1}{r}\right) \chi_{4}\right]\right\}\right|_{r=a_{4}}=0, \\
\left.\frac{2 \mu_{4}}{a_{4}}\left\{\left(\frac{\partial \Phi_{4}}{\partial r}-\frac{\Phi_{4}}{r}\right)+\left[r \frac{\partial^{2} \chi_{4}}{\partial r^{2}}+\frac{\partial \chi_{4}}{\partial r}+\left(\frac{r k_{4,2}^{2}}{2}-\frac{1}{r}\right) \chi_{4}\right]\right\}\right|_{r=a_{4}}= \\
\left.\frac{1}{\rho_{5} \omega^{2}} \frac{\partial p_{5}}{\partial r}\right|_{r=a_{4}} .
\end{gathered}
$$

At $r=a_{5}$

$$
\begin{gathered}
\left.\left\{\left[2 \mu_{6} \frac{\partial^{2} \Phi_{6}}{\partial r^{2}}-\lambda_{6} k_{6,1}^{2} \Phi_{6}\right]+2 \mu_{6}\left[\frac{\partial^{3}\left(r \chi_{6}\right)}{\partial r^{3}}+k_{6,2}^{2} \frac{\partial\left(r \chi_{6}\right)}{\partial r}\right]\right\}\right|_{r=a_{5}}=-\left.p_{5}\right|_{r=a_{5}} \\
\left.\frac{2 \mu_{6}}{a_{5}}\left\{\left(\frac{\partial \Phi_{6}}{\partial r}-\frac{\Phi_{6}}{r}\right)+\left[r \frac{\partial^{2} \chi_{6}}{\partial r^{2}}+\frac{\partial \chi_{6}}{\partial r}+\left(\frac{r k_{6,2}^{2}}{2}-\frac{1}{r}\right) \chi_{6}\right]\right\}\right|_{r=a_{5}}=0 \\
\left.\frac{2 \mu_{4}}{a_{5}}\left\{\left(\frac{\partial \Phi_{6}}{\partial r}-\frac{\Phi_{6}}{r}\right)+\left[r \frac{\partial^{2} \chi_{6}}{\partial r^{2}}+\frac{\partial \chi_{6}}{\partial r}+\left(\frac{r k_{6,2}^{2}}{2}-\frac{1}{r}\right) \chi_{6}\right]\right\}\right|_{r=a_{5}}= \\
\left.\frac{1}{\rho_{5} \omega^{2}}\left(\frac{\partial p_{5}}{\partial r}\right)\right|_{r=a_{5}}
\end{gathered}
$$


The following eigenfunctions are obtained for the spheroidal modes by substituting Equations (54) into Equations (44):

$$
\begin{aligned}
& U_{i}(r, \theta)=\left\{\begin{array}{c}
A_{4 i-3}\left[\frac{1}{r} j_{n}\left(k_{i, 1} r\right)\right]+A_{4 i-2}\left[\frac{1}{r} y_{n}\left(k_{i, 1} r\right)\right]+ \\
A_{4 i-1}\left[\frac{(n+1)}{r} j_{n}\left(k_{i, 2} r\right)-k_{i, 2} j_{n+1}\left(k_{i, 2} r\right)\right]+ \\
A_{4 i}\left[\frac{(n+1)}{r} y_{n}\left(k_{i, 2} r\right)-k_{i, 2} y_{n+1}\left(k_{i, 2} r\right)\right]
\end{array}\right\} \times \\
& {\left[-(n+1) \cot \theta P_{n}^{m}(\cos \theta)+\frac{n-m+1}{\sin \theta} P_{n+1}^{m}(\cos \theta)\right] \text {, }} \\
& V_{i}(r, \theta)=\left\{\begin{array}{c}
A_{4 i-3}\left[-\frac{m}{r \sin \theta} j_{n}\left(k_{i, 1} r\right)\right]+A_{4 i-2}\left[-\frac{m}{r \sin \theta} y_{n}\left(k_{i, 1} r\right)\right]+ \\
A_{4 i-1}\left(-\frac{m}{r \sin \theta}\right)\left[(n+1) j_{n}\left(k_{i, 2} r\right)-k_{i, 2} r j_{n+1}\left(k_{i, 2} r\right)\right]+ \\
A_{4 i}\left(-\frac{m}{r \sin \theta}\right)\left[(n+1) y_{n}\left(k_{i, 2} r\right)-k_{i, 2} r y_{n+1}\left(k_{i, 2} r\right)\right]
\end{array}\right\} \times \\
& P_{n}^{m}(\cos \theta), \\
& W_{i}(r, \theta)=\left\{\begin{array}{c}
A_{4 i-3}\left[\frac{n}{r} j_{n}\left(k_{i, 1} r\right)-k_{i, 1} j_{n+1}\left(k_{i, 1} r\right)\right]+ \\
A_{4 i-2}\left[\frac{n}{r} y_{n}\left(k_{i, 1} r\right)-k_{i, 1} y_{n+1}\left(k_{i, 1} r\right)\right]+ \\
A_{4 i-1}\left[\frac{n(n+1)}{r} j_{n}\left(k_{i, 2} r\right)\right]+A_{4 i}\left[\frac{n(n+1)}{r} y_{n}\left(k_{i, 2} r\right)\right]
\end{array}\right\} \times \\
& P_{n}^{m}(\cos \theta) \text {, } \\
& U_{5}(r, \theta)=\frac{1}{\rho_{5} \omega^{2}}\left\{A_{17}\left[\frac{1}{r} j_{n}\left(k_{5,3} r\right)\right]+A_{18}\left[\frac{1}{r} y_{n}\left(k_{5,3} r\right)\right]\right\} \times \\
& {\left[-(n+1) \cot \theta P_{n}^{m}(\cos \theta)+\frac{n-m+1}{\sin \theta} P_{n+1}^{m}(\cos \theta)\right],} \\
& V_{5}(r, \theta)=\frac{1}{\rho_{5} \omega^{2}}\left\{A_{17}\left[-\frac{m}{r \sin \theta} j_{n}\left(k_{5,3} r\right)\right]+A_{18}\left[-\frac{m}{r \sin \theta} y_{n}\left(k_{5,3} r\right)\right]\right\} \times \\
& P_{n}^{m}(\cos \theta), \\
& W_{5}(r, \theta)=\frac{1}{\rho_{5} \omega^{2}}\left\{\begin{array}{c}
A_{17}\left[\frac{n}{r} j_{n}\left(k_{5,3} r\right)-k_{5,3} j_{n+1}\left(k_{5,3} r\right)\right]+ \\
A_{18}\left[\frac{n}{r} y_{n}\left(k_{5,3} r\right)-k_{5,3} y_{n+1}\left(k_{5,3} r\right)\right]
\end{array}\right\} \times \\
& P_{n}^{m}(\cos \theta), \\
& U_{6}(r, \theta)= \\
& \left\{A_{19}\left[\frac{1}{r} j_{n}\left(k_{6,1} r\right)\right]+A_{20}\left[\frac{(n+1)}{r} j_{n}\left(k_{6,2} r\right)-k_{6,2} j_{n+1}\left(k_{6,2} r\right)\right]\right\} \times \\
& {\left[-(n+1) \cot \theta P_{n}^{m}(\cos \theta)+\frac{n-m+1}{\sin \theta} P_{n+1}^{m}(\cos \theta)\right] \text {, }}
\end{aligned}
$$




$$
\begin{gathered}
V_{6}(r, \theta)=\left\{\begin{array}{c}
A_{19}\left[\left(-\frac{m}{r \sin \theta}\right) j_{n}\left(k_{6,1} r\right)\right]+A_{20}\left(-\frac{m}{r \sin \theta}\right) \times \\
{\left[(n+1) j_{n}\left(k_{6,2} r\right)-k_{6,2} r j_{n+1}\left(k_{6,2} r\right)\right]}
\end{array}\right\} \times \\
P_{n}^{m}(\cos \theta), \\
W_{6}(r, \theta)=\left\{\begin{array}{c}
A_{19}\left[\frac{n}{r} j_{n}\left(k_{6,1} r\right)-k_{6,1} j_{n+1}\left(k_{6,1} r\right)\right]+ \\
A_{20}\left[\frac{n(n+1)}{r} j_{n}\left(k_{6,2} r\right)\right]
\end{array}\right\} \begin{array}{c}
P_{n}^{m}(\cos \theta),
\end{array}
\end{gathered}
$$

where $i=1,2,3,4$.

For the investigation of torsional modes we formulate the boundary and continuity conditions which express the balance between the tangential stresses in the solid layers as well as the equality of the corresponding tangential displacements on junctions of the layers. The outer layer is assumed to be free from tangential stress components. Using these assumptions together with Equations (53) we obtain the following boundary and continuity conditions:

At $r=a$

$$
\left.\frac{\mu_{1}}{a}\left(\frac{\partial \Psi_{1}}{\partial r}-\frac{\Psi_{1}}{r}\right)\right|_{r=a}=0 .
$$

At $r=a_{1}$

At $r=a_{2}$

$$
\begin{gathered}
\left.\frac{\mu_{1}}{a_{1}}\left(\frac{\partial \Psi_{1}}{\partial r}-\frac{\Psi_{1}}{r}\right)\right|_{r=a_{1}}=\left.\frac{\mu_{2}}{a_{1}}\left(\frac{\partial \Psi_{2}}{\partial r}-\frac{\Psi_{2}}{r}\right)\right|_{r=a_{1}}, \\
\left.\Psi_{1}\right|_{r=a_{1}}=\left.\Psi_{2}\right|_{r=a_{1}} .
\end{gathered}
$$

At $r=a_{3}$

$$
\begin{gathered}
\left.\frac{\mu_{2}}{a_{2}}\left(\frac{\partial \Psi_{2}}{\partial r}-\frac{\Psi_{2}}{r}\right)\right|_{r=a_{2}}=\left.\frac{\mu_{3}}{a_{2}}\left(\frac{\partial \Psi_{3}}{\partial r}-\frac{\Psi_{3}}{r}\right)\right|_{r=a_{2}}, \\
\left.\Psi_{2}\right|_{r=a_{2}}=\left.\Psi_{3}\right|_{r=a_{2}} .
\end{gathered}
$$

At $r=a_{4}$

$$
\begin{gathered}
\left.\frac{\mu_{3}}{a_{3}}\left(\frac{\partial \Psi_{3}}{\partial r}-\frac{\Psi_{3}}{r}\right)\right|_{r=a_{3}}=\left.\frac{\mu_{4}}{a_{3}}\left(\frac{\partial \Psi_{4}}{\partial r}-\frac{\Psi_{4}}{r}\right)\right|_{r=a_{3}}, \\
\left.\Psi_{3}\right|_{r=a_{3}}=\left.\Psi_{4}\right|_{r=a_{3}} .
\end{gathered}
$$

For the torsional modes, the eigenfunctions are as follow for $i=1,2,3,4$ :

$$
\begin{gathered}
U_{i}(r, \theta)=\left\{B_{2 i-1}\left[-\frac{m}{a \sin \theta} j_{n}\left(k_{i, 2} r\right)\right]+B_{2 i}\left[-\frac{m}{a \sin \theta} y_{n}\left(k_{i, 2} r\right)\right]\right\} \times \\
P_{n}^{m}(\cos \theta), \\
V_{i}(r, \theta)=\left\{B_{2 i-1}\left[-\frac{1}{a} j_{n}\left(k_{i, 2} r\right)\right]+B_{2 i}\left[-\frac{1}{a} y_{n}\left(k_{i, 2} r\right)\right]\right\} \times \\
{\left[-(n+1) \cot \theta P_{n}^{m}(\cos \theta)+\frac{n-m+1}{\sin \theta} P_{n+1}^{m}(\cos \theta)\right],} \\
W_{i}(r, \theta)=0 .
\end{gathered}
$$




\begin{tabular}{|l|c|c|c|c|c|}
\hline & $\lambda_{i}(\mathrm{~Pa})$ & $\mu_{i}(\mathrm{~Pa})$ & $\rho_{i}\left(\mathrm{~kg} / \mathrm{m}^{3}\right)$ & $\alpha_{i}(\mathrm{~m} / \mathrm{s})$ & $\beta_{i}(\mathrm{~m} / \mathrm{s})$ \\
\hline “Crust" & $33.4 \times 10^{9}$ & $36.4 \times 10^{9}$ & $2.6 \times 10^{3}$ & $6.40 \times 10^{3}$ & $3.75 \times 10^{3}$ \\
\hline $\begin{array}{l}\text { "Upper } \\
\text { Mantle } \\
-1 \text { " }\end{array}$ & $110 \times 10^{9}$ & $85.3 \times 10^{9}$ & $3.7 \times 10^{3}$ & $8.70 \times 10^{3}$ & $4.80 \times 10^{3}$ \\
\hline $\begin{array}{l}\text { "Upper } \\
\text { Mantle } \\
-2 \text { " }\end{array}$ & $156 \times 10^{9}$ & $130 \times 10^{9}$ & $4.0 \times 10^{3}$ & $10.2 \times 10^{3}$ & $7.50 \times 10^{3}$ \\
\hline $\begin{array}{l}\text { "Lower } \\
\text { Mantle" }\end{array}$ & $343 \times 10^{9}$ & $238 \times 10^{9}$ & $5.0 \times 10^{3}$ & $12.8 \times 10^{3}$ & $6.90 \times 10^{3}$ \\
\hline $\begin{array}{l}\text { "Outer } \\
\text { Core" }\end{array}$ & $783 \times 10^{9}$ & 0 & $11.1 \times 10^{3}$ & $8.4 \times 10^{3}$ & 0 \\
\hline $\begin{array}{l}\text { "Inner } \\
\text { Core" }\end{array}$ & $115 \times 10^{10}$ & $206 \times 10^{9}$ & $12.9 \times 10^{3}$ & $11.0 \times 10^{3}$ & $4.00 \times 10^{3}$ \\
\hline
\end{tabular}

Table 1. Physical properties of the moon's layers and the phase velocities $\alpha_{i}$ and $\beta_{i}$ of the Pand S- waves respectively.

\subsection{Example}

Consider the following fictional example: In the course of the "Avatar mission" it was found that the moon "Pandora" has a spherical layered structure with five concentric layers and an "inner core". It was determined that the outer radius of the moon is $a=6371 \mathrm{~km}$; the boundary between the upper layer ("crust") and the "upper mantle-1" is situated at radius $a_{1}=6336 \mathrm{~km}$; the boundary between the "upper mantle- 1 " and "upper mantle-2" is at radius $a_{2}=5961 \mathrm{~km}$; the boundary between the "upper mantle-2" and "lower mantle" is at radius $a_{3}=5711 \mathrm{~km}$; the boundary between the "lower mantle" and the "outer core" is at radius $a_{4}=3482 \mathrm{~km}$; and finally, the boundary between the "outer core" and "inner core" is at radius $a_{5}=1217 \mathrm{~km}$. It is assumed that the outer layers ("crust", "upper mantle-1", "upper mantle-2", "lower mantle" and the "inner core") are solids and the "outer core" is liquid, which does not support shear stresses (i.e. it is simulated by the model of an inviscid ideal fluid). Table 1 gives the physical properties of the moon as well as the phase velocities of the P-wave

$$
\alpha_{i}=\sqrt{\frac{\lambda_{i}+2 \mu_{i}}{\rho_{i}}}
$$

and the S-wave

$$
\beta_{i}=\sqrt{\frac{\mu_{i}}{\rho_{i}}}
$$

$i=1, \cdots, 6$ where $\lambda_{i}, \mu_{i}$ are Lame coefficients (keeping in mind that $\lambda_{5}=\kappa_{5}$ the bulk modulus with $\left.\mu_{5}=0\right)$ and $\rho_{i}$ are mass densities of the layers.

For the spheroidal mode of vibration, for each pair of wave numbers $n$ and $m$, by using Equations (54) and applying the boundary and continuity conditions Equations (56), $\cdots,(75)$ we obtain a matrix equation

$$
M\left(\begin{array}{c}
A_{1} \\
\vdots \\
A_{20}
\end{array}\right)=\left(\begin{array}{c}
0 \\
\vdots \\
0
\end{array}\right)
$$


where the $20 \times 20$ matrix $M=M(\omega)=\left(a_{i, j}(\omega)\right)_{i, j=1, \cdots, 20}$. Linear algebra tells us that Equation (98) has a nontrivial solution if the determinant

$$
\operatorname{det}(M(\omega))=0
$$

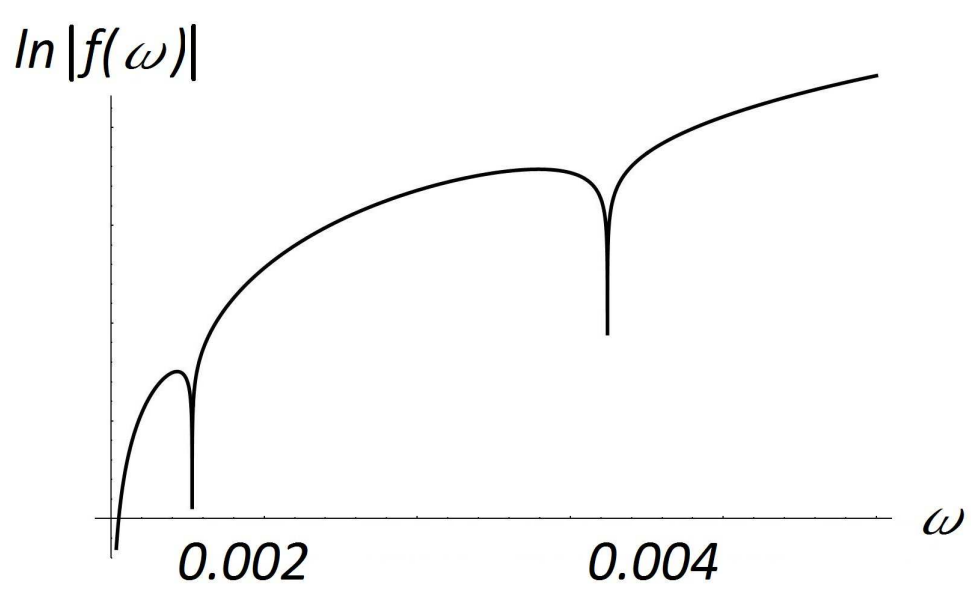

Fig. 2. A graph of the function $g(\omega)=\ln |\operatorname{det}(M(\omega))|$. The vertical "spikes" point towards roots of the function $f(\omega)=M(\omega)$. It appears that "good guesses" for the first and second eigenvalues are $\omega_{1} \approx 0.002 \mathrm{rad} . \mathrm{s}^{-1}$ and $\omega_{2} \approx 0.004 \mathrm{rad} . \mathrm{s}^{-1}$ respectively.

Determining the roots of the function

$$
f(\omega)=\operatorname{det}(M(\omega))
$$

numerically yields a sequence of eigenvalues $\omega_{1}<\omega_{2}<\cdots$ (and hence periods of vibration $T_{1}>T_{2}>\cdots$ where $T_{j}=\frac{2 \pi}{\omega_{j}}$ ). For instance, with $n=m=2$, a graph of the function

$$
g(\omega)=\ln |f(\omega)|
$$

will be similar to that given in Figure 2. The vertical "spikes" indicate roots of the function $f(\omega)$. It appears that "good guesses" for the first and second eigenvalues are $\omega_{1} \approx$ $0.002 \mathrm{rad}$. $\mathrm{s}^{-1}$ and $\omega_{2} \approx 0.004 \mathrm{rad} . \mathrm{s}^{-1}$ respectively. These "good guesses" are then used in the numerical routine of a computer algebra system, producing $\omega_{1}$ and $\omega_{2}$ to, say, four significant figures of accuracy and hence yield the values $T_{1}=\frac{2 \pi}{\omega_{1}}=68.7 \mathrm{~min}$ and $T_{2}=24.3 \mathrm{~min}$ as given in Table 2. Once a suitable number of eigenvalues have been determined, by choosing a fixed eigenvalue $\omega_{k}$, the matrix $M\left(\omega_{k}\right)$ has known constant entries. Hence it is now possible to determine a non-trivial solution to

$$
M\left(\omega_{k}\right)\left(\begin{array}{c}
A_{1} \\
\vdots \\
A_{20}
\end{array}\right)=\left(\begin{array}{c}
0 \\
\vdots \\
0
\end{array}\right) .
$$




\begin{tabular}{|c|c|c|c|c|c|c|}
\hline \multirow{2}{*}{$\mathrm{n}$} & \multirow{2}{*}{$\mathrm{m}$} & $T_{1}(\min )$ & $T_{2}(\min )$ & $T_{3}(\min )$ & $T_{4}(\min )$ & $T_{5}(\min )$ \\
& & $\eta_{1}$ & $\eta_{2}$ & $\eta_{3}$ & $\eta_{4}$ & $\eta_{5}$ \\
\hline \multirow{2}{*}{2} & \multirow{2}{*}{2} & 68.7 & 24.3 & 15.7 & 14.4 & 9.2 \\
& & -0.86 & -0.63 & -0.14 & -0.24 & -0.17 \\
\hline \multirow{2}{*}{3} & \multirow{2}{*}{2} & 41.2 & 17.7 & 13.2 & 10.3 & 7.8 \\
& & -0.47 & -0.51 & -0.05 & -0.10 & -0.13 \\
\hline \multirow{2}{*}{3} & \multirow{2}{*}{3} & 41.2 & 17.7 & 13.2 & 10.3 & 7.8 \\
& & -0.70 & -0.76 & -0.08 & -0.14 & -0.19 \\
\hline \multirow{2}{*}{4} & \multirow{2}{*}{2} & 28.2 & 14.4 & 11.8 & 7.9 & 7.1 \\
& & -0.25 & -0.43 & -0.03 & -0.04 & -0.12 \\
\hline \multirow{2}{*}{4} & \multirow{2}{*}{3} & 28.2 & 14.4 & 11.8 & 7.9 & 7.1 \\
& & -0.44 & -0.64 & -0.04 & -0.06 & -0.19 \\
\hline \multirow{2}{*}{4} & \multirow{2}{*}{4} & 28.2 & 14.4 & 11.8 & 7.9 & 7.1 \\
& & -0.58 & -0.86 & -0.05 & -0.08 & -0.25 \\
\hline \multirow{2}{*}{5} & \multirow{2}{*}{2} & 21.0 & 12.5 & 10.5 & 6.7 & 6.5 \\
& & -0.20 & -0.32 & -0.06 & -0.07 & -0.02 \\
\hline \multirow{2}{*}{5} & \multirow{2}{*}{3} & 21.0 & 12.5 & 10.5 & 6.7 & 6.5 \\
& & -0.30 & -0.48 & -0.09 & -0.11 & -0.03 \\
\hline \multirow{2}{*}{5} & \multirow{2}{*}{4} & 21.0 & 12.5 & 10.5 & 6.7 & 6.5 \\
& & -0.40 & -0.64 & -0.12 & -0.14 & -0.04 \\
\hline \multirow{2}{*}{5} & \multirow{2}{*}{5} & 21.0 & 12.5 & 10.5 & 6.7 & 6.5 \\
& & -0.50 & -0.80 & -0.15 & -0.18 & -0.05 \\
\hline
\end{tabular}

Table 2. Period of oscillation $T_{j}$ and Bryan's factor $\eta_{j}$ of the spheroidal modes of vibration for wave numbers $n$ and $m$.

Consequently the eigenfunctions given by Equations (76) to (84) are completely determined. Keeping Equations (40) and (42) in mind, Bryan's factor is calculated by using

$$
\eta=\frac{2 \int_{0}^{\pi}\left\{\sum_{i=1}^{6} \int_{a_{i}}^{a_{i-1}}\left[\rho_{i}\left(U_{i} \cos \theta+W_{i} \sin \theta\right) V_{i}\right] r^{2} d r\right\} \sin \theta d \theta}{\int_{0}^{\pi}\left\{\sum_{i=1}^{6} \int_{a_{i}}^{a_{i-1}} \rho_{i}\left(U_{i}^{2}+V_{i}^{2}+W_{i}^{2}\right) r^{2} d r\right\} \sin \theta d \theta}
$$

where $a_{0}=a$ and $a_{6}=0$.

For the spheroidal modes of vibration, the periods of oscillation $T_{j}=\frac{2 \pi}{\omega_{j}}$ as well as the corresponding Bryan's factors $\eta_{j}$ for a pair of wave numbers $m$ and $n$ are given in Table 2 .

Doing a similar analysis (as explained above) for the torsional modes, the eigenfunctions given by Equations (93), ․, (95) as well as the eigenvalues are completely determined by applying the boundary and continuity conditions given by Equations (85), - , (92) to Equations (55). Here Equation (103) for Bryan's factor may be simplified, because the radial displacements are zero $W_{i}=0$. Bryan's factor for the torsional modes is calculated by using the formula

$$
\eta_{T M}=\frac{2 \int_{0}^{\pi}\left\{\sum_{i=1}^{6} \int_{a_{i}}^{a_{i-1}} U_{i} V_{i} r^{2} d r\right\} \sin \theta \cos \theta d \theta}{\int_{0}^{\pi}\left\{\sum_{i=1}^{6} \int_{a_{i}}^{a_{i-1}} \rho_{i}\left(U_{i}^{2}+V_{i}^{2}\right) r^{2} d r\right\} \sin \theta d \theta} .
$$

Values of the periods of oscillation $T_{j}$ and the corresponding Bryan's factors $\eta_{j}$ for the $n$ and $m$ torsional modes are given in Table 3 . 


\begin{tabular}{|c|c|c|c|c|c|c|}
\hline \multirow{2}{*}{$\mathrm{n}$} & \multirow{2}{*}{$\mathrm{m}$} & $T_{1}(\min )$ & $T_{2}(\min )$ & $T_{3}(\min )$ & $T_{4}(\min )$ & $T_{5}(\min )$ \\
& & $\eta_{1}$ & $\eta_{2}$ & $\eta_{3}$ & $\eta_{4}$ & $\eta_{5}$ \\
\hline \multirow{2}{*}{2} & \multirow{2}{*}{2} & 42.4 & 11.9 & 7.2 & 5.0 & 3.7 \\
& & 0.33 & 0.33 & 0.33 & 0.33 & 0.33 \\
\hline \multirow{2}{*}{3} & \multirow{2}{*}{2} & 27.2 & 11.0 & 7.0 & 4.9 & 3.7 \\
& & 0.17 & 0.17 & 0.17 & 0.17 & 0.17 \\
\hline \multirow{2}{*}{3} & \multirow{2}{*}{3} & 27.2 & 11.0 & 7.0 & 4.9 & 3.7 \\
& & 0.25 & 0.25 & 0.25 & 0.25 & 0.25 \\
\hline \multirow{2}{*}{4} & \multirow{2}{*}{2} & 20.7 & 10.0 & 6.8 & 4.8 & 3.6 \\
& & 0.10 & 0.10 & 0.10 & 0.10 & 0.10 \\
\hline \multirow{2}{*}{4} & \multirow{2}{*}{3} & 20.7 & 10.0 & 6.8 & 4.8 & 3.6 \\
& & 0.15 & 0.15 & 0.15 & 0.15 & 0.15 \\
\hline \multirow{2}{*}{4} & \multirow{2}{*}{4} & 20.7 & 10.0 & 6.8 & 4.8 & 3.6 \\
& & 0.20 & 0.20 & 0.20 & 0.20 & 0.20 \\
\hline \multirow{2}{*}{5} & \multirow{2}{*}{2} & 16.9 & 9.1 & 6.5 & 4.7 & 3.5 \\
& & 0.07 & 0.07 & 0.07 & 0.07 & 0.07 \\
\hline \multirow{2}{*}{5} & \multirow{2}{*}{3} & 16.9 & 9.1 & 6.5 & 4.7 & 3.5 \\
& & 0.10 & 0.10 & 0.10 & 0.10 & 0.10 \\
\hline \multirow{2}{*}{5} & \multirow{2}{*}{4} & 16.9 & 9.1 & 6.5 & 4.7 & 3.5 \\
& & 0.13 & 0.13 & 0.13 & 0.13 & 0.13 \\
\hline \multirow{2}{*}{5} & \multirow{2}{*}{5} & 16.9 & 9.1 & 6.5 & 4.7 & 3.5 \\
& & 0.17 & 0.17 & 0.17 & 0.17 & 0.17 \\
\hline
\end{tabular}

Table 3. Period of oscillation $T_{j}$ and Bryan's factor $\eta_{j}$ of the torsional modes of vibration for wave numbers $n$ and $m$.

\section{Conclusions and discussions}

After deriving expressions for Bryan's factor $\eta$ in terms of eigenvalues and eigenfunctions of vibration we demonstrated that neither Bryan's effect nor the value of Bryan's factor $\eta$ depend on the inclusion of light, isotropic, viscous damping in the model. Consequently we neglected damping in the model that we presented. However, we pointed out that preliminary studies indicate that the inclusion of "impurities" such as mass-stiffness, prestress and anisotropic damping into the model appears to produce "capture effects" such as the rotation angle of the vibration pattern varying periodically. Hence more realistic models (as opposed to ideal situations) that will include these "impurities" need to be developed.

Solutions were obtained for the dynamic equations of slowly rotating, vibrating planets or moons considered as ideal elastic solid and inviscid acoustic bodies composed of, for instance, four solid layers, one liquid layer and a solid core. Boundary conditions were formulated for calculating the eigenvalues and eigenfunctions of vibration and a brief discussion of how they are numerically calculated was given. It was pointed out that for each pair of wave numbers $n$ and $m$, there is a sequence of eigenvalues $\omega_{1}<\omega_{2}<\cdots$ and hence periods of vibration $T_{1}>T_{2}>\cdots\left(\right.$ where $\left.T_{j}=\frac{2 \pi}{\omega_{j}}\right)$.

The model derived indicates that there are two modes of vibration, namely the "spheroidal" and "torsional" modes and Bryan's effect occurs for both modes.

Table 1 provides feasible physical properties for a fictional moon and gives possible phase velocities for both $\mathrm{P}$ - and S-waves. 
Using Table 1, a numerical experiment produced Table 2, where it appears that for the spheroidal modes, for a given polar wave number $n$ and a given non-negative integer $j$, the period of oscillation $T_{j}$ does not depend on the circumferential wave number $m$. However, Bryan's factor $\eta$ depends on both wave numbers $n$ and $m$ as well as the period of oscillation $T_{j}$ for each pair $n$ and $m$. Bryan's factor $\eta$ appears to be negative in all cases and this means that an antinode of vibration will rotate through the moon's crusts in the opposite direction to the moon's rotation at a rate $\eta \varphi$, where $\varphi$ is the moon's rotation rate.

Another numerical experiment produced Table 3, where it appears that for torsional modes, for a given wave number $n$ and a given non-negative integer $j$, the period of oscillation $T_{j}$ is independent of the circumferential wave number $m$ and that it varies with $n$. However, Bryan's factor $\eta$ appears to be invariant and independent of the period of oscillation for a given pair of wave numbers $n$ and $m$, but appears to vary as each pair varies and it appears to be positive in all cases. Consequently Bryan's factors for the torsional modes depend only on angular components of the vibrating patterns, that is, on the corresponding wave numbers $n$ and $m$, and not on radial vibrations because all radial displacements $W_{i}=0$. These positive Bryan's factors $\eta$ indicate that the antinodes of vibration will rotate through the moon's crusts in the same direction as the moon's rotation at a rate $\eta \gamma$, where $\gamma$ is the moon's rotation rate.

\section{Acknowledgement and disclaimer}

This material is based upon work supported financially by the Tshwane University of Technology (TUT), the Council for Scientific and Industrial Research (CSIR) of South Africa and the National Research Foundation (NRF) of South Africa (NRF grant reference number EV2009011 400005). Any opinions, findings and conclusions or recommendations expressed in this material are those of the authors and therefore the TUT, the CSIR and the NRF do not accept any liability in regard thereto.

\section{References}

Bryan, G.H. (1890). On the beats in the vibrations of a revolving cylinder or bell, Proceedings of the Cambridge Philosophical Society 7, pp. 101-111.

Eringen, A.E. \& Suhubi, E.S. (1975). Elastodynamics, vol. I1. Academic Press, New York.

Goldstein, H., Poole, C. \& Safko, J. (2001). Classical mechanics, 3rd ed, Addison-Wesley, Reading, MA.

Loveday, P.W \& Rogers, C.A. (1998). Free vibrations of elastically supported thin cylinders including gyroscopic effects, Journal of Sound and Vibration 217(3), pp. 547-562.

Nayfeh, A.H. (1973). Perturbation methods, John Wiley \& Sons, Inc., New York.

Rayleigh, J.W.S. (1894). The theory of sound, volume I, second edition, MacMillan \& Company, London.

Redwood, M. (1960). Mechanical waveguides, Pergamon Press, Oxford.

Rozelle, D.M. (2009). The hemispherical resonator gyro: From wineglass to the planets, Proceedings of the 19th AAS/AIAA Space Flight Mechanics Meeting, February 8-12, Savannah, Georgia, USA, pp. 1157-1178.

Shatalov, M.Y., Joubert, S.V., Coetzee, C.E. \& Fedotov, I. (2009). Free vibration of rotating hollow spheres containing acoustic media, Journal of Sound and Vibration, 322(4-5), pp. 1038-1047.

Available online: doi:10.1016/j.jsv.2008.11.020, accessed July 2011. 
Shatalov, M.Y., Joubert, S.V. \& Coetzee, C.E. (2011). The influence of mass imperfections on the evolution of standing waves in slowly rotating spherical bodies, Journal of Sound and Vibration, 330, pp. 127-135.

Available online: doi:10.1016/j.jsv.2010.08.001, accessed July 2011.

Spiegel, M.R. (1967). Theoretical mechanics. McGraw-Hill, New York.

Zhuravlev, V. \& Klimov, D. (1988). Applied methods in the theory of oscillations. Moscow: Nauka (in Russian). 


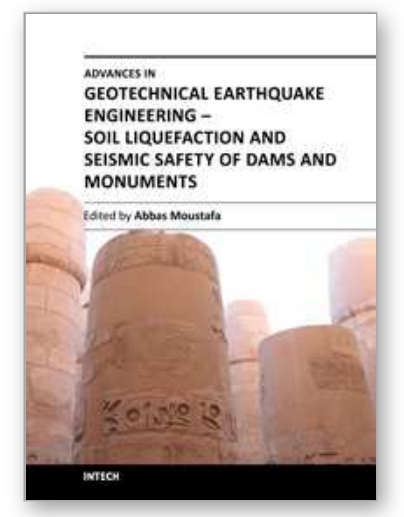

\author{
Advances in Geotechnical Earthquake Engineering - Soil \\ Liquefaction and Seismic Safety of Dams and Monuments \\ Edited by Prof. Abbas Moustafa
}

ISBN 978-953-51-0025-6

Hard cover, 424 pages

Publisher InTech

Published online 10, February, 2012

Published in print edition February, 2012

This book sheds lights on recent advances in Geotechnical Earthquake Engineering with special emphasis on soil liquefaction, soil-structure interaction, seismic safety of dams and underground monuments, mitigation strategies against landslide and fire whirlwind resulting from earthquakes and vibration of a layered rotating plant and Bryan's effect. The book contains sixteen chapters covering several interesting research topics written by researchers and experts from several countries. The research reported in this book is useful to graduate students and researchers working in the fields of structural and earthquake engineering. The book will also be of considerable help to civil engineers working on construction and repair of engineering structures, such as buildings, roads, dams and monuments.

\title{
How to reference
}

In order to correctly reference this scholarly work, feel free to copy and paste the following:

Michael Y. Shatalov, Stephan V. Joubert and Charlotta E. Coetzee (2012). The Vibration of a Layered Rotating Planet and Bryan's Effect, Advances in Geotechnical Earthquake Engineering - Soil Liquefaction and Seismic Safety of Dams and Monuments, Prof. Abbas Moustafa (Ed.), ISBN: 978-953-51-0025-6, InTech, Available from: http://www.intechopen.com/books/advances-in-geotechnical-earthquake-engineering-soil-liquefactionand-seismic-safety-of-dams-and-monuments/-the-vibration-of-a-layered-rotating-planet-and-bryan-s-effect

\section{INTECH}

open science | open minds

\author{
InTech Europe \\ University Campus STeP Ri \\ Slavka Krautzeka 83/A \\ 51000 Rijeka, Croatia \\ Phone: +385 (51) 770447 \\ Fax: +385 (51) 686166 \\ www.intechopen.com
}

\author{
InTech China \\ Unit 405, Office Block, Hotel Equatorial Shanghai \\ No.65, Yan An Road (West), Shanghai, 200040, China \\ 中国上海市延安西路65号上海国际贵都大饭店办公楼 405 单元 \\ Phone: +86-21-62489820 \\ Fax: +86-21-62489821
}


(C) 2012 The Author(s). Licensee IntechOpen. This is an open access article distributed under the terms of the Creative Commons Attribution 3.0 License, which permits unrestricted use, distribution, and reproduction in any medium, provided the original work is properly cited. 